\title{
Parkinsonism Related to Brain Tumors: A Case Report and Review of the Literature
}

\author{
Takao Yasuhara*, Takashi Agari, Hirokazu Kambara, Tomotsugu Ichikawa, Kazuhiko Kurozumi, \\ Shigeki Ono, Yasuyuki Miyoshi, Koji Tokunaga and Isao Date
}

Department of Neurological Surgery, Okayama University Graduate School of Medicine, Dentistry, and Pharmaceutical Sciences, Okayama, 700-8558, Japan

\begin{abstract}
Parkinsonism related to brain tumor is reviewed with the presentation of our case. Neurosurgeons or neurologists know that some patients with brain tumor present parkinsonism, although further enlightenment is required. The mechanisms are divided into 6 subgroups, 1. direct oppression to the basal ganglia; 2. distortion and stress to the midbrain; 3. neuronal loss in the substantia nigra; 4. involvement of the basal ganglia; 5. damage of the fiber connection between the basal ganglia and supplementary motor area; 6. old age. In the recent 11 years, 16 cases were reported. Pediatric cases, localized cases with relatively small lesion and post-irradiation cases were increasingly reported. The prognoses of brain tumor-related parkinsonism varied and independent of the pathology, location, and therapies of the tumor. All we can do is to select the appropriate therapy including surgical removal of the tumor by the correct estimation of the damaged region.
\end{abstract}

Keywords: Basal ganglia, brain stem, pediatric brain tumor, parkinsonism.

\section{INTRODUCTION}

The cardinal symptoms of Parkinson's disease are akinesia, rigidity, resting tremor and impairment of the postural reflex. As widely understood, parkinsonism is divided into idiopathic parkinsonism including Parkinson's disease and symptomatic parkinsonism with causative diseases. In this article, our case of parkinsonism related to brain tumor is presented with review of the literature.

\section{CASE PRESENTATION}

\section{Our Case with Dystonia after Multimodal Therapy for Germ Cell Tumor at the Pineal Region}

A 29-year-old man admitted to our institute for the movement disorder of eyes and gait disturbance. CT and MRI demonstrated heterogenously enhanced mass at the pineal region with involvement in the right substantia nigra (Fig. 1A). The patient received endoscopic biopsy and subsequently diagnosed as germ cell tumor (germinoma). Three courses of chemotherapy using Ifosfamide, carboplatin and etoposide were performed. Then irradiation to the whole brain at $30 \mathrm{~Gy}$ and local irradiation at $24 \mathrm{~Gy}$ were added. The tumor was completely controlled with no enhanced lesion in Gd-T1 weighted image of MRI after another course of chemotherapy, although the scar remained at the right midbrain (Fig. 1B). On the course of chemotherapy, the patient received endoscopic third ventriculostomy for hydrocephalus. At 2 months after discharge, dystonia with rigidity of the left elbow and hand appeared. Medication using dopamine,

\footnotetext{
*Address correspondence to this author at the Department of Neurological surgery, Okayama University Graduate School of Medicine, Dentistry, and Pharmaceutical Sciences, 2-5-1, Shikata-cho, Okayama, 700-8558, Japan; Tel: 81-86-235-7336; Fax: 81-86-227-0191;

E-mail: tyasu37@cc.okayama-u.ac.jp
}

efficient effects on the symptoms. Thalamotomy was performed after our diagnosis that the tumor damaged the dopaminergic neuronal systems at the substantia nigra and the surrounding structures, even though the tumor was controlled. The rigidity of his elbow was moderately ameliorated by the surgery, although the symptoms of his hand were not improved at all. Additionally, we tried the electrical stimulation for the left forearm at low frequency. However, the symptoms of his hand were minimally improved. For 2 years, the ADL of the patient was stable with the impaired hand (KPS: 70), although the tumor was completely controlled.

\section{DISCUSSION}

\section{Historic Background of Brain Tumor and Parkin- sonism}

After the case report of brain tumor with parkinsonism was initially reported in 1953 [1], several reports and reviews were followed. In 1997, Kondo and colleagues reported an excellent review paper entitled as 'brain tumor and parkinsonism' [2]. They demonstrated that the number of reports on brain tumor and parkinsonism might decrease, although there were many reports before publication of their initial review. The reason might be the publicity of parkinsonism induced by brain tumor among clinicians and the early diagnosis of brain tumor before manifestation of parkinsonism by development of the diagnostic imaging technique. Between 1997 and 2008, only 16 cases were reported for 11 years (Table 1). Pediatric cases, localized cases and post-irradiation cases were increasingly reported for recent 11 years. At the same time, as recognized in previous reports, large brain tumors such as frontal astrocytoma and meningioma of adult might constitute a majority including unreported cases. 

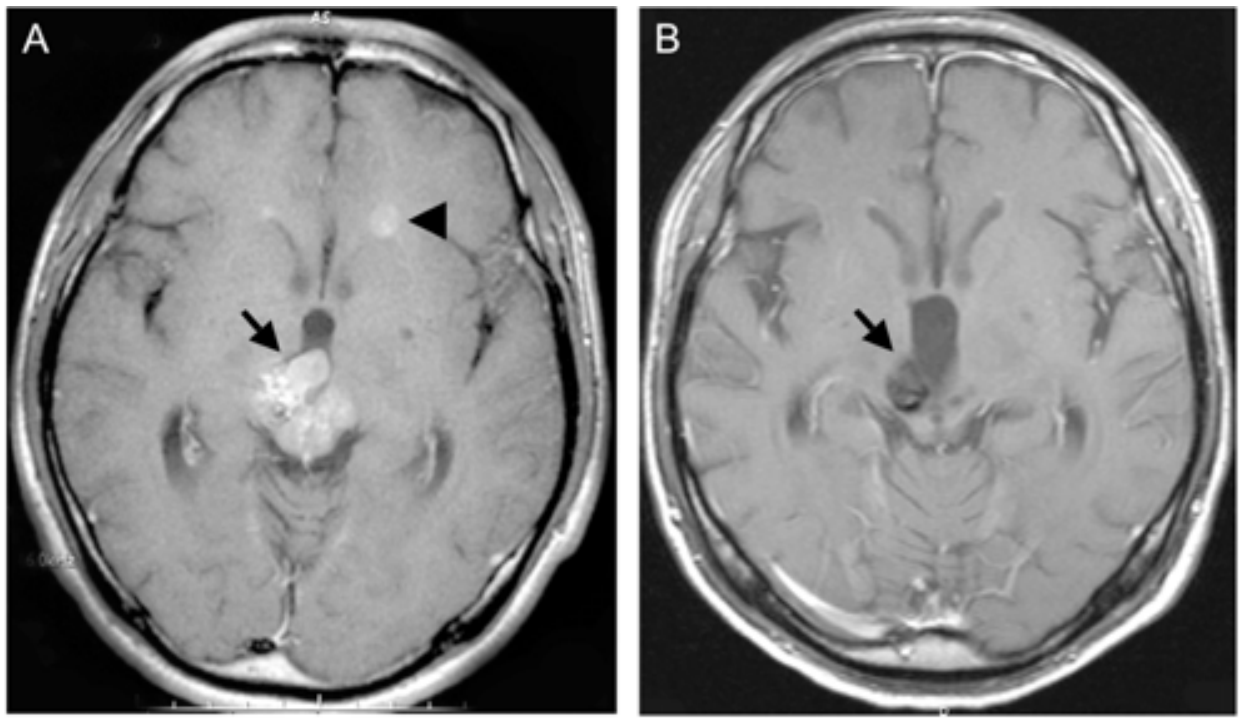

Fig. (1). Germ cell tumor at the pineal region.

A: Gd-enhanced T1-weighted image of MRI demonstrates the heterogenously enhanced lesion (arrow) with the longitudinal extension from the pineal region and involvement in the right substantia nigra. A small round lesion with slight enhancement (arrowhead) was also found at the anterior horn of the left lateral ventricle.

B: Gd-enhanced T1-weighted image of MRI demonstrates the non-enhanced small lesion (arrow) with the involvement in the right substantia nigra.

Table 1. A Summary of Case Reports about Parkinsonism Related to Brain Tumor in the Recent 11 Years

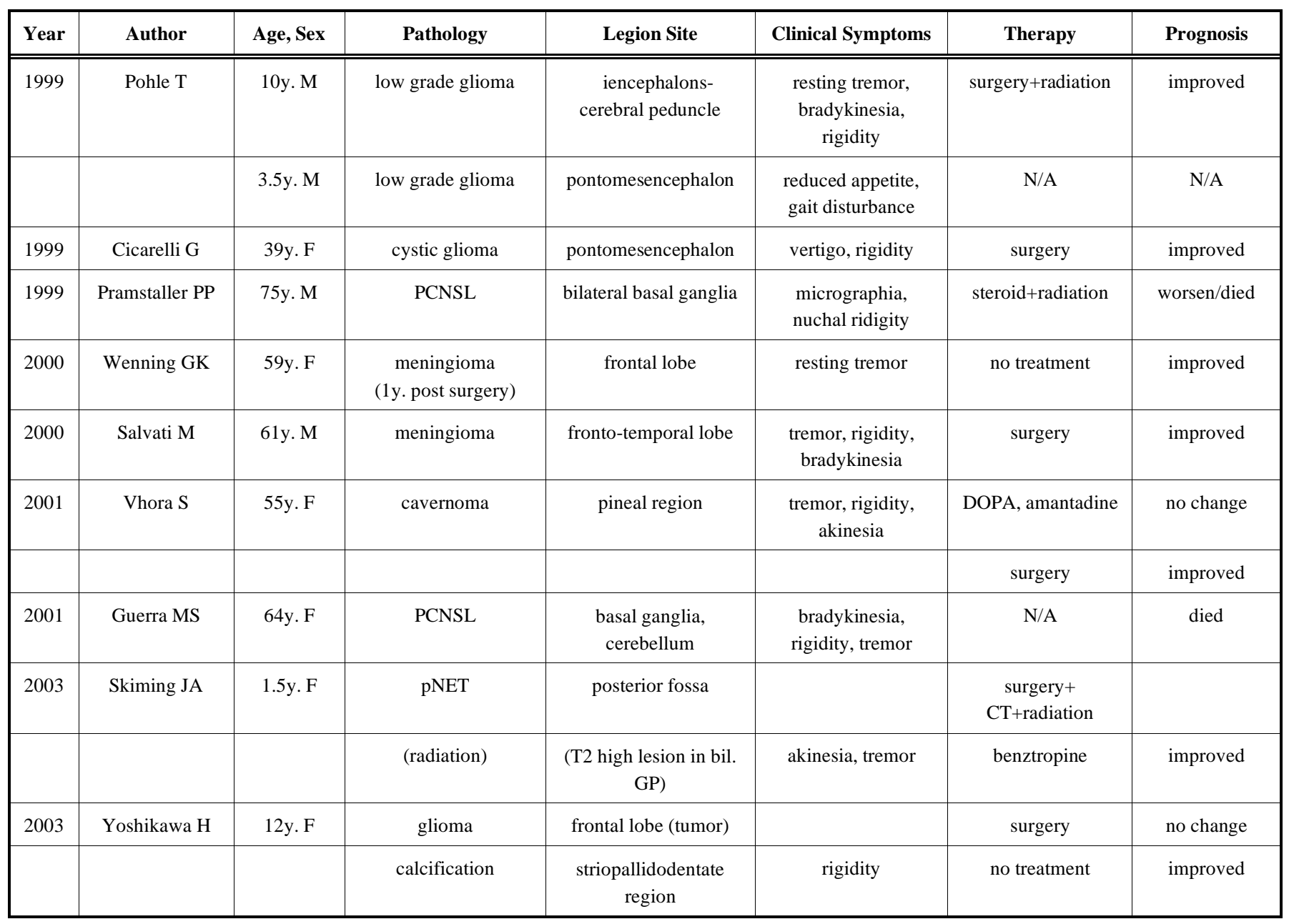


(Table 1). Contd.....

\begin{tabular}{|c|c|c|c|c|c|c|c|}
\hline Year & Author & Age, Sex & Pathology & Legion Site & Clinical Symptoms & Therapy & Prognosis \\
\hline \multirow[t]{3}{*}{2006} & Voermans NC & $14 \mathrm{y} . \mathrm{F}$ & craniopharyngioma & seller region & hypokinesia, rigidity & surgery+radiation & \\
\hline & & & (radiation) & $\begin{array}{l}\text { (T2 high lesion in bil. } \\
\text { GP) }\end{array}$ & & L-DOPA & $\begin{array}{c}\text { a little } \\
\text { improved }\end{array}$ \\
\hline & & & & & & & $\begin{array}{l}\text { then, } \\
\text { spontaneously } \\
\text { improved }\end{array}$ \\
\hline 2007 & Burkhardt K & $85 y . M$ & pilocytic astrocytoma & brain stem & N/A & N/A & N/A \\
\hline 2007 & Asada $\mathrm{T}$ & 70y. M & gliomatosis cerebri & $\begin{array}{c}\text { bilateral } \\
\text { fronto-parietal lobe }\end{array}$ & $\begin{array}{l}\text { frozen gait, tremor, } \\
\text { rigidity }\end{array}$ & anti-convulsant & worsened \\
\hline
\end{tabular}

The age of cases varied from 1.5 to 85 years old. The most common histology was glioma. Tumors usually localized at the basal ganglia or brain stem. Almost all cases presented several symptoms of the cardinal symptoms of parkinsonism. The selected therapies and responses also varied, which indicated that appropriate therapies can not be selected by the histology or the location of the tumor. PCNSL: primary central nervous system lymphoma, GP: globus pallidus, N/A: not applicable.

\section{Symptoms of Parkinsonism Induced by Brain Tumor}

As shown in the table, most of the cases presented more than one symptoms of Parkinson's disease. In the review paper by Kondo [2], 44\% (32/73 cases) of the patients with brain tumor presenting parkinsonism began at only parkinsonism and the half began at parkinsonism with or without other symptoms. Taking into account 16 cases found for recent 11 years, $63 \%$ (56/89 cases) of the patients initially manifested parkinsonism, which indicated that cases presenting parkinsonism as an initial symptom might be increasingly reported. In association with this, half of the cases, reported recently (8/16 cases) had a relatively small lesion, which directly affected nigrostriatal systems or nucleus in the circuit of the basal ganglia. In consideration of these facts, the cases of a large brain tumor with brain edema, presenting initially other neurological deficits followed by parkinsonism might decrease in number.

\section{Pathology of Brain Tumors which Cause Parkinsonism}

As described in the previous section, large meningiomas or astrocytomas were frequently reported. The pathology of the recent 16 cases were 8 gliomas (including 2 gliomatosis cerebri) [3-10], 2 meningiomas [11, 12], 2 malignant lymphomas [13, 14], 2 angiomas [15, 16], 1 craniopharyngioma [17], 1 primitive neuroectodermal tumor (PNET) [18]. A case of giomatosis cerebri was misdiagnosed and treated as multiple system atrophy, because of the unusual clinical manifestation including extreme old age, parkinsonism as an initial symptom, and the lack of high intensity area extending into the white matter in FLAIR and T2-weighted images of MRI. The necropsy revealed the diffuse cell infiltration into the whole brain including the substantia nigra. This case might be a good lesson, although it might be very rare [4].

\section{Treatment and Prognosis of Parkinsonism Induced by Brain Tumor}

In order to assess the appropriate therapy and the reliable prognosis of parkinsonism induced by brain tumor, the oc- currence mechanisms should be considered. Kondo and colleagues propounded 6 reasonable mechanisms of it: 1 . direct oppression to the basal ganglia of the tumor itself; 2. distortion and stress to the midbrain; 3 . neuronal loss in the substantia nigra by the tumor invasion; 4 . involvement of the basal ganglia by the deep-seated tumor; 5 . damage of the fiber connection between the basal ganglia and supplementary motor area; 6 . old age. In case of direct oppression to the basal ganglia of the tumor itself or damage of fiber connection between the basal ganglia and supplementary motor area, tumor removal and subsequent relief of the disease state might ameliorate symptoms. Practically, a case of large meningioma [12] or transient encephalopathy with brain edema after irradiation [18] might hold true. On the other hand, cases by distortion and stress to the midbrain or neuronal loss in the substantia nigra by the tumor invasion might respond well to the replacement of dopamine theoretically. However in the recent reports, the medication using dopamine exerted almost no or weak therapeutic effects on many cases with parkinsonism. The reason might be the severe and irreversible impairment at the initiation of the medication. Furthermore, the therapeutic effects on cases with involvement of the basal ganglia by the deep-seated tumor might depend on whether the function of dopamine receptor was preserved or not. Anyway, the early diagnosis and the exploration for the appropriate therapy including surgical removal might critically determine the outcomes of the patients. Additionally, severe prognoses of PCNSL patients with parkinsonism should be noted.

\section{Characteristics of Tumor-Related Parkinsonism in Pediatrics}

Parkinsonism in pediatrics is rare. Brain tumors as well as cerebral ischemia and trauma might also cause parkinsonism. The prognosis is generally good if the primary disease is correctly treated with the relief of symptoms at the acute stage [19]. In recent reports, 3 cases demonstrated remarkable amelioration of symptoms in the 4 cases under 14 years old $[3,6,17,18]$. At 6 months after irradiation, 2 cases 
with PNET and craniopharyngioma presented parkinsonism temporarily with high intensity lesion in T2-weighted image of MRI at the globus pallidum. The former ameliorated the symptoms with the administration of anti-cholinergic agent at 7 months after irradiation and the latter spontaneously resolved symptoms at 9 months after irradiation.

\section{CONCLUSIONS}

We reviewed the previous reports about parkinsonism related to brain tumor with the presentation of our related case. Recently, brain tumor is recognized as a reason for movement disorders including Parkinson's disease for neurosurgeons and neurologists. Further enlightenment is required for clinicians to prevent delayed diagnosis and wrong therapy for cases of brain tumor with movement disorders, although the effective therapy and prognosis might differ in each case. Unfortunately some cases are difficult to be treated finely. At now, we need to select the appropriate therapy for the patients by estimating the damaged region immediately.

\section{REFERENCES}

[1] Margulies ME. Parkinsonism and brain tumor. J Nerv Ment Dis 1953; 117: 550-2.

[2] Kondo T. Brain tumor and parkinsonism. Nippon Rinsho 1997; 55: $118-22$.

[3] Pohle T, Krauss JK. Parkinsonism in children resulting from mesencephalic tumors. Mov Disord 1999; 14: 842-6.

[4] Molho ES. Gliomatosis cerebri may present as an atypical Parkinsonian syndrome. Mov Disord 2004; 19: 341-4.

[5] Cicarelli G, Pellecchia MT, Maiuri F, et al. Brain stem cystic astrocytoma presenting with "pure" parkinsonism. Mov Disord 1999; 14: $364-6$
[6] Yoshikawa H, Abe T. Transient parkinsonism in bilateral striopallidodentate calcinosis. Pediatr Neurol 2003; 29: 75-7.

[7] Yoshimura M, Yamamoto $\mathrm{T}$, Iso-o $\mathrm{N}$, et al. Hemiparkinsonism associated with a mesencephalic tumor. J Neurol Sci 2002; 197: 89-92.

[8] Asada T, Takayama Y, Tokuriki Y, et al. Gliomatosis cerebri presenting as a parkinsonian syndrome. J Neuroimaging 2007; 17: 269-71.

[9] Burkhardt K, Heuberger F, Delavelle J. Pilocytic astrocytoma in the elderly. Clin Neuropathol 2007; 26: 306-10.

[10] Ho Bl, Lieu AS, Hsu CY. Hemiparkinsonism secondary to an infiltrative astrocytoma. Neurologist 2008; 14: 258-61.

[11] Wenning GK, Luginger E, Sailer U, et al. Postoperative parkisonian tremor in a patient with a frontal meningioma. Mov Disord 1999; 14: 366-8.

[12] Salvati M, Frati A, Ferrari P, et al. Parkinsonian syndrome in a patient with a pterional meningioma: case report and review of the literature. Clin Neurol Neurosurg 2000; 102: 243-5.

[13] Pramstaller PP, Salerno A, Bhatia K, et al. Primary central nervous system lymphoma presenting with a parkinsonian syndrome of pure akinesia. J Neurol 1999; 246: 934-8.

[14] Sanchez-Guerra M, Cerezal L, Leno C, et al. Primary brain lymphoma presenting as Parkinson's disease. Neuroradiology 2001; 43: 36-40.

[15] Vhora S, Kobayashi S, Okudera H. Pineal cavernous angioma presenting with parkinsonism. J Clin Neurosci 2001; 8: 263-66.

[16] Ertan S, Gulcin B, Tanriverdi T, et al. Parkinsonism caused by cavernoma located in basal ganglion. Parkinsonism Relat Disord 2005; 11: 517-9.

[17] Voermans NC, Bloem BR, Janssens G, et al. Secondary parkinsonism in childhood: a rare complication after radiotherapy. Pediatr Neurol 2006; 34: 495-8.

[18] Skiming JA, McDowell HP, Wright N, et al. Secondary parkinsonism: an unusual late complication of craniospinal radiotherapy given to a 16-month child. Med Pediatr Oncol 2003; 40: 132-134.

[19] Pranzatelli MR, Mott SH, Pavlakis SG, et al. Clinical spectrum of secondary parkinsonism in childhood: a reversible disorder. Pediatr Neurol 1994; 10: 131-40.

(c) Yasuhara et al.; Licensee Bentham Open.

This is an open access article licensed under the terms of the Creative Commons Attribution Non-Commercial License (http://creativecommons.org/licenses/by-nc/3.0/) which permits unrestricted, non-commercial use, distribution and reproduction in any medium, provided the work is properly cited. 